\title{
Network Topology Determination using Least Absolute Value State Estimation
}

\author{
H. Singh \\ Member \\ F.L. Alvarado \\ Fellow \\ Department of Electrical and Computer Engineering \\ The University of Wisconsin, Madison WI 53706
}

\begin{abstract}
In energy control centers, the estimates of the system state can often be affected by errors in the assumptions made about the topology of the network. This paper introduces a technique that avoids making incorrect assumptions about network status by estimating the status of suspect lines as a part of the state estimation process. This is done by introducing additional variables and measurements in the conventional state estimation problem. The success of the method relies on properties of the Least Absolute Value (LAV) estimator (an estimator that minimizes the $\ell_{1}$ norm of the measurement residuals). The performance of the method is demonstrated on standard test systems.

Keywords: WLAV estimators, L1 norms, network topology errors, power system state estimation, linear programming.
\end{abstract}

\section{Introduction}

Real-time monitoring of power systems in energy control centers requires the processing of analog measurements and status measurements. Analog measurements include line flows, bus injections and voltage magnitudes. These measurements are processed by the state estimator. These measurements may be further classified as telemetered, pseudo or virtual measurements depending on their nature. Status measurements describe the status of circuit breakers in the system and are used by a topology processor to determine the realtime configuration of network elements [4]. Topology processors based on tree-search algorithms have been proposed [14,29] and are in use today. Any error in status data results in a misconfiguration of network elements (a "topology error") and can affect the results of the state estimator significantly. Furthermore, an un-

94 SM 506-6 PWRS A paper recommended and approved by the IEEE Power System Engineering Committee of the IEEE Power Engineering Society for presentation at the IEEE/PES 1994 Summer Meeting, San Francisco, CA, July $24-28$, 1994. Manus cript submitted December 29, 1993; made available for printing June 1, 1994. detected topology error can lead to serious errors when operators assess the security of a system for subsequent contingencies. This paper deals with methods which seek to avoid the occurrence of topology errors.

A number of methods have been suggested to deal with topology error detection and identification. Most methods use state estimation results to detect topology errors $[8,11,24,33]$, while some advocate rule based approaches that do not rely on state estimation [31]. This paper uses the state estimator to determine the configuration of suspect elements in the network. Instead of viewing the problem as one of detection and identification of topology errors, it is viewed as one of topology determination for network elements whose status is unknown or suspect. This is made possible by expanding the set of variables and measurements in the conventional formulation of power system state estimation. The success of the method relies on the properties of the LAV estimator. LAV estimators have been proposed as an alternative to Weighted Least Squares (WLS) state estimators due to their resistance to bad data (outliers) in the measurements $[17,19,21]$. These estimators are able to reject outliers as long as these are not leverage points $[5,6,16,17,22]$. Computationally efficient techniques to implement LAV estimators using both the simplex and interior point methods of linear programming have been proposed $[1,3,7,10,15,30]$. Related ideas have been proposed for modelling of circuit breakers in WLS state estimation [26].

\section{Effects of Topology errors}

The network elements that can be misconfigured include transmission lines, transformers, and shunt elements. The resulting topology error may involve one or multiple network elements leading to single or multiple topology errors. Topology errors can also be classified as inclusion or exclusion errors depending on whether a network element that is in operation is assumed to be out of operation or vice versa. Multiple topology errors can include both inclusion and exclusion errors [11,33].

The admittance $y_{k m}$ between any two nodes $k$ and $m$ of an electric power network is considered as a known complex parameter which is non-zero if and only if there exists a physical connection (such as a transmission line or a transformer) between the two nodes. In large net- 
works, most of the admittances $y_{k m}$ are zero, since for any given node, physical connections exist only with a few other nodes of the network. Thus, the admittance matrix of the network is a sparse matrix. Any error in the assumptions of the existing physical connections amounts to an error in the admittance matrix of the network. This error is reflected as an error in the Jacobian matrix used in state estimation [33]. This can be seen by considering the measurement model used in state estimation

$$
z=h^{\text {actual }}(x)+\epsilon
$$

where $z$ is the given $m$ dimensional measurement vector, $x$ is the unknown $n$ dimensional state vector and $\epsilon$ is the $\boldsymbol{m}$ dimensional vector of measurement noise. $h^{\text {actual }}$ is the non-linear function that relates the measurements to the states for the actual system configuration. Since it is expressed in terms of the network admittances, the function $h$ used for an assumed network topology can be different from $h^{\text {actual }}$. Thus

$$
h^{\text {actual }}(x)=h^{\text {assumed }}(x)+\Delta h(x) .
$$

The last two equations can be used to re-write the measurement model as

$$
z=h^{\text {assumed }}(x)+[\Delta h(x)+\epsilon] .
$$

The measurements that are related to a topology error have a non-zero $\Delta h_{i}(x)$. (For a topology error in branch $k-m$, the related measurements are shown in Fig. 1.) The LAV estimator would normally reject these measurements. Examination of the residuals would identify topology errors. ${ }^{1}$ However, such an approach is subject to failure if the related measurements are not rejected. This can happen in the case of zero injection measurements and also in the presence of leverage points in the state estimation model $[5,6,22]$. This paper introduces an alternative approach to solve the problem.

\section{Problem Formulation}

Consider the parameterization of every branch admittance $y_{k m}$ by a real parameter $\alpha_{k m}$. The effective branch admittance between nodes $k$ and $m$ is $y_{k m} \cdot \alpha_{k m}$ where $\alpha_{k m}$ is determined as follows:

1. $\alpha_{k m}=1$ if there exists a connection between nodes $k$ and $m$ under the given operating conditions (i.e. the line is "in service"),

2. $\alpha_{k m}=0$ if there does not exist any connection between nodes $k$ and $m$ under the given operating conditions (i.e. the line is "out of service"), or

\footnotetext{
${ }^{1}$ Data validation in substations using such an approach has been suggested in [20].
}

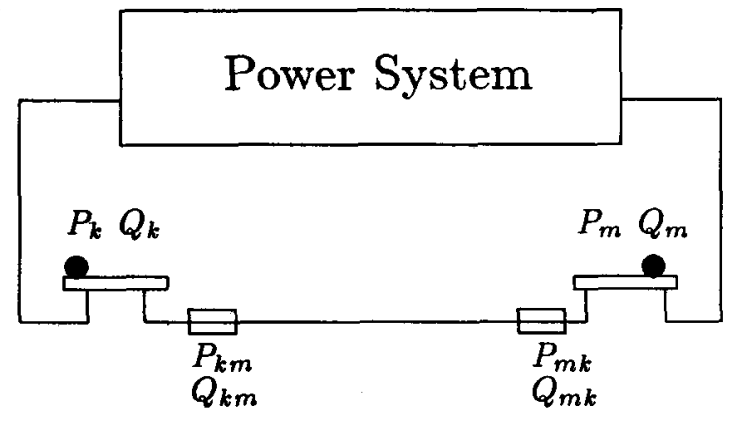

Fig. 1: Related flow and injection measurements for branch $k-m$.

3. $\alpha_{k m}=0.5$ if the status of the connection between nodes $k$ and $m$ is uncertain. The value of 0.5 is chosen as an initial guess in the estimation of all $\alpha_{k m}$ corresponding to lines with unknown status. The total number of such lines is $p$.)

The objective is to estimate all parameters $\alpha_{k m}$ which do not have a known value of either 0 or 1 . This is done as a part of the state estimation process. The problem is set up as follows:

$$
\min \|r\|_{1}
$$

subject to

$$
\begin{gathered}
r=z-h(x, \alpha) \\
\alpha_{i}=0 \text { or } 1 \quad \forall i=1, \ldots, p
\end{gathered}
$$

where,

$z$ is the $m$ dimensional measurement vector

$\alpha$ is the $p$ dimensional parameter vector

$x$ is the $n$ dimensional state vector $(n+p<m)$

$r$ is the $m$ dimensional vector of measurement errors

$h$ is the $m$ dimensional vector of non-linear functions which relate measurements to $x$ and $\alpha$.

The form of the functions $h$ can be found in any standard text on power systems, e.g. [13].

Although, the above problem is a non-differentiable, non-linear, mixed integer programming problem, its solution is obtained by solving a sequence of linear programming problems. Consider the relaxation of the above problem obtained by discarding the integrality constraints (6) requiring $\alpha_{i}$ to be 0 or 1 . The most common class of methods for solving the resulting nonlinear $\ell_{1}$ norm problem is Gauss-Newton [18]. The problem reduces to solving a sequence of linear $\ell_{1}$ norm problems. The properties of the LAV estimator can be used to enforce integer (or near integer) values for $\alpha$ by introducing the following additional restrictions (referred to as "0-1 pseudo-measurements")

$$
\alpha+u=z_{0}
$$




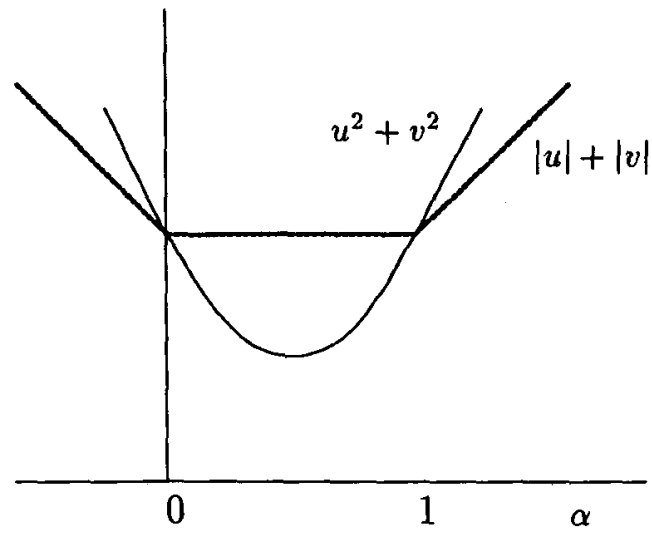

Fig. 2: Variation of the $\ell_{1}$ norm of the residuals $u$ and $v$ with $\alpha$ shows that the $0-1$ pseudo-measurements are equivalent to introducing an exact penalty function on $\alpha$ as shown by the piecewise linear function above. The minimization of the $\ell_{2}$ norm of the residuals $u$ and $v$ has a different effect as shown by quadratic function.

$$
\alpha+v=z_{1}
$$

where $z_{0}$ is a vector of zeros and $z_{1}$ is a vector of ones. These may be interpreted as conflicting pseudomeasurements for the same quantity. The premise of the paper is that the LAV estimator will choose (or give priority to) the more accurate of these measurements from every pair of $0-1$ pseudo-measurements. Fig. 2 shows the $\ell_{1}$ and $\ell_{2}$ norms of $u$ and $v$ as a function of a scalar $\alpha$. The augmented measurement vector $\tilde{z}$ can be written as $\tilde{z}^{T}=\left[z, z_{0}, z_{1}\right]^{T}$, the augmented residual vector $\tilde{r}$ is given as $[r, u, v]^{T}$ and the augmented state vector $\tilde{x}^{T}=[x, \alpha]^{T}$. The linearized LAV problem can be written as

$$
\min \|\tilde{H} \Delta \tilde{x}-\Delta \tilde{z}\|_{1}
$$

where $\tilde{H}$ is the $(m+2 p) \times(n+p)$ Jacobian matrix, $\Delta \tilde{z}$ is the $(m+2 p) \times 1$ residual vector and $\Delta \tilde{x}$ is the unknown $(n+p) \times 1$ update to the augmented state vector. The structure of the Jacobian matrix $\tilde{H}$ is

$$
\tilde{H}=\left(\begin{array}{cc}
H & A \\
0 & I \\
0 & I
\end{array}\right)
$$

where $H=\partial h / \partial x, A=\partial h / \partial \alpha$, both evaluated at $\left(x^{k}, \alpha^{k}\right)$, the values of $x$ and $\alpha$ at the current iteration.

The objective function in the above problem is a piecewise linear convex function. A general technique for optimizing such functions uses the concept of minimization models [25] as explained in the appendix. The problem is re-written as follows

$$
\begin{array}{r}
\min \zeta \\
\text { subject to } \zeta \quad \geq \quad \tilde{H} \Delta \tilde{x}-\Delta \tilde{z}
\end{array}
$$

where $\zeta$ is a $(m+2 p) \times 1$ vector. At the solution, each element of $\zeta$ is bounded below by the larger of the corresponding elements from $\tilde{H} \Delta \tilde{x}-\Delta \tilde{z}$ and $\Delta \tilde{z}-\tilde{H} \Delta \tilde{x}$. This model is an inner model.

An equivalent minimization model can be obtained by introducing slack variables to convert the inequalities in the inner model to equalities followed by addition and.subtraction of the resulting equations. Alternatively this can be obtained directly by substituting $\tilde{r}=\eta-\rho$ as $^{2}$

$$
\min e^{T}(\eta+\rho)
$$

subject to

$$
\begin{aligned}
\left(\begin{array}{lll}
\tilde{H} & I & -I
\end{array}\right)\left(\begin{array}{c}
\Delta \tilde{x} \\
\eta \\
\rho
\end{array}\right)=(\Delta \tilde{z}) \\
\eta, \rho \geq 0
\end{aligned}
$$

where $e$ is a $(m+2 p) \times 1$ vector of ones. The problem is solved for $\Delta \tilde{\boldsymbol{x}}$. The value of $\tilde{\boldsymbol{x}}$ is then updated, $\tilde{x}^{k+1} \leftarrow$ $\tilde{x}^{k}+\Delta \tilde{x}$. The constraints are linearized at the updated operating point, $\tilde{x}^{k}=\left[x^{k}, \alpha^{k}\right]^{T}$ and the procedure is repeated until convergence. The problem is similar to the conventional LAV state estimation problem and can be solved using specialized techniques that have been applied for that purpose $[1,3,7,10,15,30]$.

\section{Test Results}

The method proposed in this paper has been tested on the 6 bus Ward and Hale system and the IEEE 14, 30 and 118 bus systems. The methodology of testing starts with a power flow run for a certain known network configuration to generate the measurement set for the state estimator. This measurement set is used to estimate $\alpha$ for all the unmetered lines in the system. The results for the different systems are shown in Tables 1-4. Both, LAV and Least Squares estimates are shown for the cases studied. The presence of 0 1 pseudo-measurements on $\alpha$ helps the LAV estimator and works against the Least Squares estimator. However, in the absence of these constraints, it may not even be possible to solve the Least Squares case due to ill-conditioning of the Jacobian matrix. This is due to the small magnitudes of some elements in the columns of $A$. For example, in the 30 bus system for a given configuration and state, the condition number of the Jacobian matrix is improved from 10000 to 230 by introducing additional rows corresponding to $0-1$ measurements. The only additional computational effort involved in the method is due to the slightly expanded

\footnotetext{
"The variables $\eta$ and $p$ are subject to a "complementarity" condition $\left(\eta_{i} \rho_{i}=0\right.$ ). This condition is not explicitly written in the model since it is automatically satisfied at the solution of the minimization problem. The same cannot be said for a maximization problem. The dual formulation used in [30] can be obtained from-both the above minimization models.
} 
size of the Jacobian matrix. The measurement redundancy level used is between 2 and 2.37. The test results show that the estimates obtained for $\alpha$ are very close to 0 or 1 for the LAV estimator while they are close to 0.5 for the Least Squares estimator.

Table 1: Estimated values of $\alpha$ for the 6 bus system.

\begin{tabular}{|c|c|c|c|}
\hline Unmetered & Actual & \multicolumn{2}{|c|}{ Estimates for $\alpha_{k m}$} \\
\cline { 3 - 4 } Line & Status & LAV & Least Squares \\
\hline $1-4$ & In & 0.974 & 0.538 \\
\hline $3-4$ & In & 0.992 & 0.543 \\
\hline $4-6$ & Out & 0.023 & 0.510 \\
\hline
\end{tabular}

Table 2: Estimated values of $\alpha$ for the 14 bus system.

\begin{tabular}{|c|c|c|c|}
\hline Unmetered & Actual & \multicolumn{2}{|c|}{ Estimates for $\boldsymbol{\alpha}_{\boldsymbol{k}}$} \\
\cline { 3 - 4 } Line & Status & LAV & Least Squares \\
\hline $1-2$ & In & 1.000 & 0.561 \\
\hline $2-4$ & In & 1.000 & 0.516 \\
\hline $4-5$ & In & 0.931 & 0.518 \\
\hline $4-9$ & Out & 0.000 & 0.492 \\
\hline
\end{tabular}

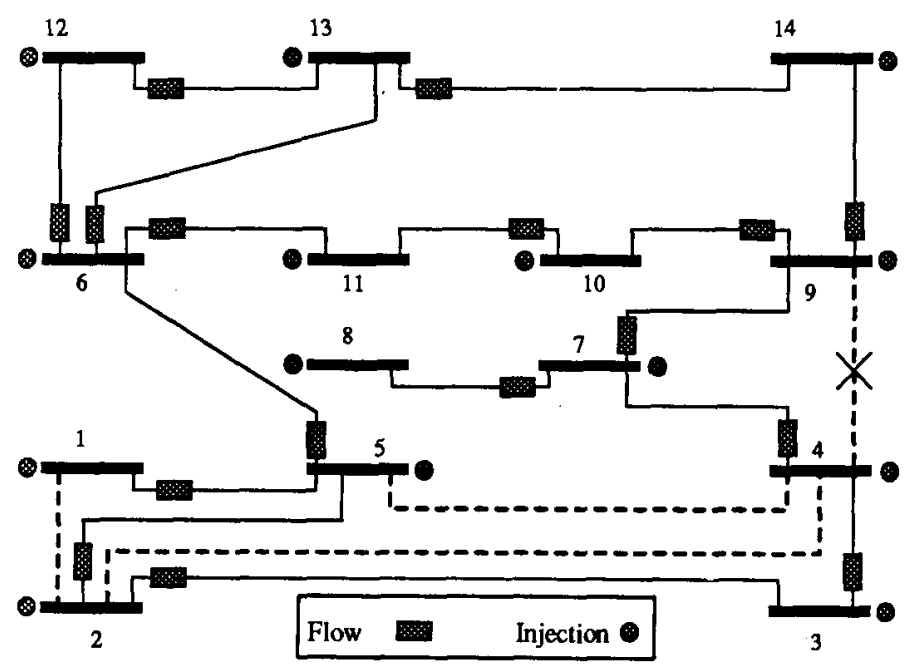

Fig. 3: IEEE 14 bus system with four unmetered lines (broken lines) whose status is suspect. Three of these lines are in service while line $4-9$ is out of service.

\section{Implementation Issues}

\section{Detectability of topology errors}

In the absence of any related injection measurements, a topology error cannot be detected by conventional means $^{3}$. Conditions for detectability of topology errors

\footnotetext{
${ }^{3}$ It is possible to detect the status of a line in the absence of related measurements, but this requires the use of more than one state estimate [2].
}

Table 3: Estimated values of $\alpha$ for the 30 bus system.

\begin{tabular}{|c|c|c|c|}
\hline Unmetered & \multirow{2}{*}{$\begin{array}{c}\text { Actual } \\
\text { Line }\end{array}$} & \multicolumn{2}{|c|}{ Estimates for $\alpha_{k m}$} \\
\cline { 3 - 4 } & Status & LAV & Least Squares \\
\hline $1-2$ & In & 0.999 & 0.608 \\
\hline $8-28$ & Out & 0.000 & 0.499 \\
\hline $12-15$ & In & 0.999 & 0.501 \\
\hline $19-20$ & In & 0.995 & 0.500 \\
\hline $22-24$ & In & 0.995 & 0.500 \\
\hline $29-30$ & Out & 0.001 & 0.499 \\
\hline
\end{tabular}

Table 4: Estimated values of $\alpha$ for the 118 bus system.

\begin{tabular}{|c|c|c|c|}
\hline Unmetered & Actual & \multicolumn{2}{|c|}{ Estimates for $\alpha_{k m}$} \\
\cline { 3 - 4 } Line & Status & LAV & Least Squares \\
\hline $4-5$ & In & 1.000 & 0.509 \\
\hline $4-11$ & In & 1.000 & 0.534 \\
\hline $5-11$ & In & 1.000 & 0.548 \\
\hline $14-15$ & In & 0.946 & 0.499 \\
\hline $15-19$ & In & 1.000 & 0.505 \\
\hline $25-27$ & In & 1.008 & 0.668 \\
\hline $44-45$ & In & 1.000 & 0.509 \\
\hline $114-115$ & In & 1.000 & 0.499 \\
\hline
\end{tabular}

have been addressed in $[8,9,33]$. Test results presented in this paper assume that these conditions are satisfied. In the absence of related measurements, the column of $A$ that corresponds to the given branch is all zeros and results in making $A$ rank deficient. The presence of 0-1 pseudo-measurements for $\alpha$ ensures that, even in the absence of related measurements, the matrix $\tilde{H}$ is not rank deficient. Although algorithms for calculation of LAV estimates do not necessarily require $\tilde{H}$ to be full rank, it is an assumption made in many interior point methods (such as those used in [30]) and is helpful numerically. However, estimates of $\alpha$ obtained without related measurements may not be reliable.

\section{Increasing the redundancy level}

The ability of the LAV estimator to reject outliers among the measurements depends upon the presence of a sufficient number of good measurements, i.e., a reasonable redundancy level. It is possible to improve the redundancy level by exploiting the fact that, although the state vector and the measurements change with time, the parameters do not. Hence, a sequence of measurements can be processed together to improve the redundancy. If two scans are chosen, the following system is obtained (subscripts in parenthesis denote time instants):

$$
\left(\begin{array}{ccc}
H_{(1)} & 0 & A_{(1)} \\
0 & H_{(2)} & A_{(2)} \\
0 & 0 & I \\
0 & 0 & I
\end{array}\right)\left(\begin{array}{c}
\Delta x_{(1)} \\
\Delta x_{(2)} \\
\Delta \alpha
\end{array}\right)=\left(\begin{array}{c}
\Delta z_{(1)} \\
\Delta z_{(2)} \\
\Delta z_{1} \\
\Delta z_{0}
\end{array}\right)
$$


This approach assumes that a network switching does not take place between the chosen measurement samples. Similar ideas have been expressed for parameter estimation using a recursive least squares approach $[12,23]$. Methods to solve the LAV problem by processing small subsets of measurements at a time using Lagrangian relaxation have been developed recently [32].

\section{Optimality Conditions}

The original problem has been solved by relaxing the integrality constraints requiring $\alpha_{i}$ to be 0 or 1 . The feasible region of the resulting relaxation contains the feasible region of the original problem. The optimal solution for the relaxed problem is also optimal for the problem if and only if it is feasible for the original problem, i.e. $\alpha$ is 0 or 1 . There is no guarantee that all components of $\alpha$ will be integer ${ }^{4}$. Although the properties of LAV estimators make it reasonable to expect integer (or near integer) estimates for $\alpha$ in the relaxed problem, this may not always be the case, particularly in the presence of bad data among the measurements.

The method to deal with a non-integer estimate for $\alpha_{i}$ is to introduce the restriction $\alpha_{i}=0$ or $\alpha_{i}=1$. The resulting non-convex problem can then be solved using a branch and bound approach [18]. A branch and bound approach was used for the more general problem of bad data detection in [27]. In the problem considered here, only a small subset (possibly empty) of $\alpha$ variables may need to be branched upon. This makes the problem tractable for large systems.

\section{Conclusions}

The paper has introduced a new method for determining the topology of electric power networks by introducing status variables in the non-linear $\ell_{1}$ norm problem. This amounts to introducing additional variables and constraints in conventional LAV state estimation. Test results from standard systems indicate that that the method can make it possible to estimate the status of suspect lines with only minor modifications to existing LAV estimators.

\section{Acknowledgement}

This paper is based on work supported by NSF grant ECS-9215271.

\section{References}

[1] A. Abur and M. K. Celik. A fast algorithm for the weighted least absolute value state estimation. IEEE Transactions on Power Systems, 6(1), February 1991.

${ }^{4}$ There are instances when it is possible to predict that the solution of a relaxed problem will be integer, e.g. when the constraint matrix is totally unimodular [28]. However, this is difficult to do here.
[2] F.L. Alvarado. Determination of external system topology errors. IEEE Transactions on Power Appara tus and Systems, 100(11):4553-4561, November 1981.

[3] E.W. Bacon, K.A. Clements, and P.W. Davis. Accelerated interior point methods for least absolute value state estimation in electric power networks. In Athens Power Tech, Joint International Conference NTUAIEEE/PES, September 1993.

[4] A. Bose and K.A. Clements. Real time modelling of power networks. Proceedings of the IEEE, 75(12):16071622, December 1987.

[5] M. K. Çelik and A. Abur. A robust WLAV state estimator using transformations. IEEE Transactions on Power Systems, 7(1):106-113, February 1992.

[6] M. K. Celik and A. Abur. Use of scaling in WLAV estimation of power system states. IEEE Transactions on Power Systems, 7(2):684-692, May 1992.

[7] K. A. Clements, P. W. Davis, and K. D. Frey. An efficient algorithm for computing the weighted least absolute value estimage in power system static state estimation. In Proceedings of the IFAC Conference on Power Systems and Power Plant Control, 1989.

[8] K.A. Clements and P.W. Davis. Detection and identification of topology errors in electric power systems. IEEE Transactions on Power Apparatus and Systems, 3(4):1748-1753, 1988.

[9] K.A. Clements and P.W. Davis. Fundamental results in the detection and identification of topology errors in electric power networks. Internal Report, Worcestor Poly technic Institute, 1992.

[10] K.A. Clements, P.W. Davis, and K.D. Frey. An interior point algorithm for weighted least absolute value power ssytem state estimation. IEEE Transactions on Power Systems, 1991. To appear. Presented at the IEEE/PES Winter Meeting, paper 91-WM 235-2 PWRS.

[11] A. Simões Costa and J.A. Leão. Identification of topology errors in state estimation. IEEE Transactions on Power Systems, 8(4), November 1993.

[12] A.S. Debs. Estimation of steady state model parameters. IEEE Transactions on Power Apparatus and Systems, 93(5):1260-1268, September/October 1974.

[13] A.S. Debs. Modern Power Systems Control and Operation. Kluwer Academic Publishers, Norwell, Massachussetts, 1988 .

[14] T.E. DyLiacco, K.A. Ramarao, and A.W. Weiner. Network status analysis for real time systems. In $8^{\text {th }}$ PICA Conference Proceedings, pages 356-362, July 1973.

[15] A. A. El-Keib and H. Singh. Fast linear programming state estimation using the dual formulation. IEEE Transactions on Power Systems, 7(3):1088-1097, 1992.

[16] S.P. Ellis and S. Morgenthaler. Leverage and breakdown in $\mathrm{L}_{1}$ regression. Journal of the American Statistical Association, 87(417):143-148, March 1992.

[17] D. M. Falcão, P. A. Cooke, and A. Brameller. Linear programming state estimation: Error analysis and gross error identification. IEEE Transactions on Power Systems, 3(3):809-815, August 1988.

18] R. Fletcher. Practical Methods of Optimization. Wiley, Chichester, 1987.

[19] M. R. Irving, R. C. Owen, and M. J. H. Sterling. Power system state estimation using linear programming. Proceedings of the Instution of Electrical Engineers (Part C), 125:978-985, September 1978. 
[20] M.R. Irving and M.J.H. Sterling. Substation data validation. Proceedings of the Instution of Electrical Engineers (Part C), 129:119-122, May 1982.

[21] W. W. Kotiuga and M. Vidyasagar. Bad data rejection properties of weighted least absolute value techniques applied to static state estimation. IEEE Transactions on Power Systems, 101(4):844-851, April 1982.

[22] V. Phaniraj L. Mili and P.J. Rousseeuw. Least median of squares estimation in power systems. IEEE Transactions on Power Systems, 6(2):511-523, 1991.

[23] W.H.E. Liu, F.F. Wu, and S.M. Lun. Estimation of parameter errors from measurement residuals in state estimation. IEEE Transactions on Power Systems, $7(1): 81-88$, February 1992.

[24] R.L. Lugtu, D.F. Hackett, K.C. Liu, and D.D. Might. Power system state estimation: Detection of topology errors. IEEE Transactions on Power Apparatus and Systems, 99(6):2406-2411, November 1981.

[25] R.R. Meyer, M.V. Thakkar, and W.P. Hallman. Rational mixed-integer and polyhedral union minimization models. Mathematics of Operations Research, 5(1):135-146, February 1980.

[26] A. Monticelli. Modelling circuit breakers in weighted least squares state estimation. IEEE Transactions on Power Systems, 8(3), August 1993.

[27] A. Monticelli, F.F. Wu, and M. Yen. Multiple bad data identification for state estimation by combinatorial optimization. IEEE Transactions on Power Delivery, PWRD-1(3):361-369, July 1986.

[28] Christos H. Papadimitriou and Kenneth Steiglitz. Combinatorial Optimization: Algorithms and Complexity. Prentice Hall, Englewood Cliffs, 1982.

[29] A. M. Sasson, S. T. Ehrman, P. Lynch, and L. S. VanSlyck. Automatic power system network topology determination. IEEE Transactions on Power Apparatus and Systems, 92:610-618, March/April 1973.

[30] H. Singh and F.L. Alvarado. WLAV state estimation using interior point methods. IEEE Transactions on Power Systems, 9(3):1478-1484, August 1994.

[31] N. Singh and H. Glavitsch. Detection and identification of topological errors in online power system analysis. IEEE Transactions on Power Systems, 6(1):324331, February 1991.

[32] M.G. Sklar and R.D. Armstrong. Lagrangian approach for large scale least absolute value estimation. Computers in Operations Research, 20(1):83-93, 1993.

[33] F.F. Wu and W.H.E. Liu. Detection and identification of topology errors by state estimation. IEEE Transactions on Power Systems, 4(1):176-183, February 1989.

\section{Appendix}

\section{Minimization Models}

The basic idea of a minimization model is to replace a given function by a minimization problem in terms of other functions which are of a simpler form. To state this more formally, let $f: \Re^{n} \rightarrow \Re$ be a real function and $S$ be a subset of $\Re^{n}$. Let $M M(x)$ denote the following optimization problem (where $x$ is a parameter):

$$
\min \{g(y) \mid y \in Y \cap G(x)\}
$$

where $y \in \Re^{m}, Y \subseteq \Re^{m}$, and $G(x)$ is a subset of $\Re^{m}$ for any $x$. Let $\omega(x)$ be the optimal value for $M M(x)$. Then $M M(x)$ is said to be a minimization model for $f(x)$ on $S$ if $f(x)=\omega(x)$ for all $x \in S$.

The application of minimization models is based on a theorem [25] which says that when a function appearing as a term in the objective function of a minimization problem has a minimization model, then the term may be replaced by its minimization model. This is of use only if the minimization model is in terms of functions that are simpler than the original function. Piecewise linear convex functions are polyhedral functions and are known to possess Linear Programming Minimization Models (LPMM)s.

As an example, the absolute value function $y=|x|$ can be represented by the following minimization model which does not involve the absolute value function

$$
y=\{\min z \mid z \geq x ; \quad z \geq-x\}
$$

A generalization of this is used to re-express the $\ell_{1}$ nórm problem as a linear program.

Fernando L. Alvarado (F'93) obtained a BS degree from the National University of Engineering in Lima, Peru, a MS degree from Clarkson University, and a Ph.D. from the University of Michigan. He is currently a Professor at the University of Wisconsin in Madison in the Department of Electrical and Computer Engineering. His main interests are in computer applications to power systems and large sparse matrix problems.

Harmohan Singh (S'87) received a BE(Hons) degree from Panjab University, India in 1987, an MS degree from The University of Alabama in 1990, and a Ph.D. from the University of Wisconsin-Madison in 1994, all in electrical engineering. His research interests include power systems analysis and mathematical programming. Harry is also interested in technical issues related to re-structuring efforts in the electric utility industry. He worked with the Pacific Gas and Electric Company during the summers of 1992 and 1994 and is a member of IEEE, SIAM, Eta Kappu Nu and Sigma Xi. 


\section{Discussion}

D.M. Vinod Kumar (Indian Institute of Technology - Kanpur, India) The authors are to be congratulated for a new approach to determine the topology of a power network. The discusser appreciates the authors response on the following:

1. Is the algorithm suitable for implementation in energy control centers for real-time operations. If so, what are the CPU times for the test system results?

2. Is the approach suitable for determining parameter errors also?

3. In [30] the WLAV method is used for the computation of state estimates. Why did the authors choose the LAV method for determining network topology?

4. The authors did not take care while writing the references. For example, in [11] page numbers (pp. 1531-1538) are not specified and in [24] the IEEE Transactions on Power Systems are mentioned as IEEE Transactions on Power Apparatus and Systems.

Manuscript received August 22, 1994.

H. Singh and F. Alvarado (University of Wisconsin-Madison) The authors thank the discusser for his interest in their paper. The points raised by the discusser are addressed as follows:
1. The computational performance of various LAV algorithms for power system state estimation has been previously addressed in $[1,3,7,10,15,30]$. Any of these algorithms can be used to implement the method presented in this paper. However, the computational effort is likely to be slightly higher than the conventional WLAV formulation. The additional effort is due to two factors: (1) the additional parameters to be estimated, and (2) the additional 0-1 pseudo-measurements. The second factor can slow down the convergence of the method on account of the large residuals that become inherent in the problem [18]. Since the number of such pseudo-measurements increases with the number of parameters to be estimated, the first factor also becomes important. This issue needs to be considered carefully in any real implementation.

2. Topology errors are an extreme case of parameter errors. The paper illustrates the LAV estimator's properties to be very useful (compared to the WLS estimator) for this extreme case.

3. The LAV and the WLAV estimator differ only in the choice of measurement weights. In order to increase the chances of obtaining integer estimates for the parameters, the $0-1$ pseudomeasurements may be given higher weights than other measurements. In that case, the method would be WLAV instead of $\mathrm{LAV}$.

4. The authors thank the discusser for correcting the references.

Manuscript received October 25, 1994. 